\title{
The sociocultural dimension in leprosy vaccine trials
}

\author{
S KARTIKEYAN, R M CHATURVEDI \& M G DEO \\ ICRC Anti-Leprosy Vaccine Project, Cancer Research Institute, \\ Tata Memorial Centre, Parel, Bombay-400012, India
}

Accepted for publication 25 August 1989

\begin{abstract}
Summary This paper briefly describes organizational, operational, and sociocultural aspects of the phase-III clinical trials of the ICRC anti-leprosy vaccine in Maharashtra, India. Our experience is that vaccine trials can be launched quickly and more cost effectively by using the services of health personnel from the existing public health infrastructure. That is why the trials could be launched in just 4 months after receiving the financial grant from the Indian Council of Medical Research, New Delhi (India). At the community level, a person-toperson approach in Health Education scores over audio-visual aids and the mass media. The compliance in target groups is increased when preventive programmes are backed-up by curative services and when their privacy and daily routine are not disturbed.
\end{abstract}

\section{Introduction}

More than 100 years after the discovery of the causative organisms, Mycobacterium leprae, leprosy continues to be a major public health problem in many developing countries. Although a number of effective drugs are now available, non-compliance in drug therapy and the emergence of drug resistant $M$. leprae are two major stumbling blocks on the road to control of the disease. Global attempts are being made to evolve a strategy for immunoprophylaxis of leprosy through the development of a vaccine.

In 1979, a 'candidate' anti-leprosy vaccine was prepared at the Cancer Research Institute, Bombay (India), using gamma-radiation killed ICRC bacilli. ${ }^{1}$ The organism, a slow growing mycobacterium, probably belonging to $M$. avium intracellulare complex, exhibits antigenic cross reactivity with $M$. leprae. This is the scientific basis for their use in the vaccine preparation.

The vaccine induces lepromin conversion in about $55 \%$ of multibacillary (MB) leprosy patients, and in $95 \%$ of the lepromin negative individuals. The conversion in some patients is associated with tissue bacillary clearance and even reversal reaction. ${ }^{1,2}$ The immune conversion is stable for about 5 years. ${ }^{3}$ The data indicate that the vaccine could be used both for immunotherapy, as an adjunct to drugs, and immunoprophylaxis. The 
vaccine was cleared for large scale phase-III trials by the Drug Controller of India in 1984. Till then it had been tried only on multibacillary (MB) leprosy patients and on healthy lepromin negative individuals in urban settings where medical facilities were readily available. In this trial the vaccine would be administered to healthy volunteers, irrespective of their lepromin status, in far flung villages where health facilities are not fully developed. This could be a major operational constraint. Therefore, a pilot study (extended phase-II incorporating feasibility of this large scale phase-III trials) was carried out in Palghar and Satpati near Bombay (Figure 1) under field conditions. ${ }^{4}$ It was obvious that if the volunteers were asked to report to the village clinic, the compliance would be low. The pilot study revealed the pattern of life in the rural areas and the best time for vaccination. As it is given as a single dose, its acceptability is high.

Hypersensitivity to $M$. leprae antigen, to which residents of an endemic area are continuously exposed, has been implied in the pathogenesis of neural lesions in leprosy. ${ }^{5,6}$ It was feared that vaccination may lead to nerve damage in lepromin positive individuals. Another purpose of the Satpati studies was therefore to investigate adverse effects, if any, of the vaccine, especially in lepromin positive contacts. But no untoward effects have been observed in any group during the last 5 years. ${ }^{4}$ Further, it was observed that the ICRC vaccine does not stimulate formation of $M$. leprae specific antibodies, ${ }^{4}$ which have been implicated in hypersensitivity reactions and in formation of immune complexes that might suppress cell mediated immunity, ${ }^{5,6}$ the dominant host defence against $M$. leprae.

This paper describes the available options, study design, operational and sociocultural aspects of this trial and how various problems were overcome.

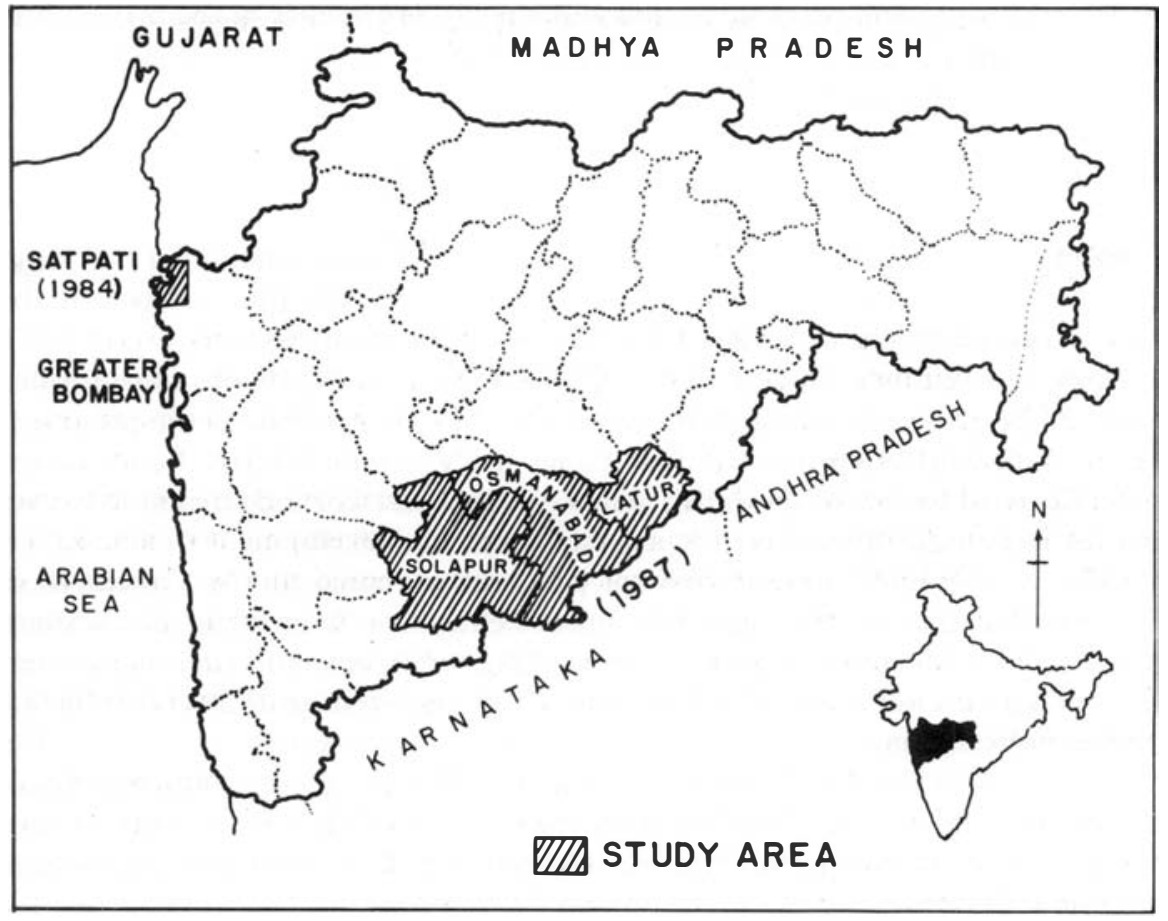

Figure 1. Map of the trial area. Figures shown in parentheses indicate the year of initiating the studies. 
Table 1. Profile of the trial area

\begin{tabular}{llll}
\hline \multicolumn{1}{c}{ Features } & \multicolumn{1}{c}{ Solapur } & Osmanabad & \multicolumn{1}{c}{ Latur } \\
\hline 1. ${ }^{*}$ Estimated mid-year $(1987)$ population $\left(1 \times 10^{6}\right)$ & $2 \cdot 19$ million & $1 \cdot 18$ million & $1 \cdot 38$ million \\
2. Area $\left(\mathrm{km}^{2}\right)$ & 15,017 & $7369 \cdot 5$ & $7264 \cdot 9$ \\
3. Population density $\left(\right.$ per $\left.\mathrm{km}^{2}\right)(1981)$ & 174 & 136 & 177 \\
4. Proportion of rural population $(1981)$ & $70 \cdot 79 \%$ & $84 \cdot 61 \%$ & $54 \cdot 61 \%$ \\
5. Literacy (1981) & $40 \cdot 68 \%$ & $35 \cdot 36 \%$ & $34 \cdot 62 \%$ \\
6. ${ }^{* *}$ Prevalence of leprosy per 1000 population $(1987)$ & $5 \cdot 8 *$ & $9 \cdot 3$ & $10 \cdot 9$ \\
7. ${ }^{* *}$ Total number of registered leprosy patients $(1987)$ & 12,795 & 10,968 & 15,164 \\
\hline Date of launching the vaccination programme & 3 Feb. 1987 & 8 June 1987 & 12 Oct. 1987 \\
\hline
\end{tabular}

Source: District Census Handbook (1981 census) Series-12 compiled by Maharashtra Census Directorate, Bombay (India). Published in 1986.

* Only certain talukas of Solapur were taken up for the trials. Prevalence in these areas was 8.5/1000.

** Based on figures provided by Leprosy Eradication Bureau, National Leprosy Eradication Programme (NLEP), Government of Maharashtra.

\section{The trial area}

The trials were launched in February 1987 in the Southeastern part of Maharashtra, India. The project area includes 3 districts-Solapur, Osmanabad and Latur-on the south-eastern fringe of Maharashtra state (Figure 1), where the prevalence of leprosy is between 8 and 10 per 1000 . The total population in the project area is about 5 million. The geographic and sociodemographic profile and leprosy prevalence in the project area are delineated in Table 1.

\section{Organizational aspects}

The Indian Council of Medical Research, New Delhi (India) sanctioned the project in October 1986. A major challenge was that the trials had to be initiated within about 4 months. The Government of Maharashtra gives a high priority to leprosy control. It is a vertical programme that is looked after, at the head office of the Health Directorate, by a very senior officer with the rank of Joint Director. In the rural areas the programme is implemented through Leprosy Control Units (LCUs) which have, besides qualified medical officers, a number of specially trained paramedical workers designated as leprosy technicians (LTs). Each unit covers a population of 200,000 to 400,000. LCUs are responsible for the entire leprosy control programme, consisting of treatment of the patients, identification of new cases, and leprosy education to increase the awareness of the disease. They also generate epidemiological data on leprosy through regular periodic house-to-house surveys. Each LT is responsible for a population of 20,000 to 25,000 . He regularly conducts village leprosy clinics, and even makes frequent home visits. He is in constant touch with the community, with whom, over the years, he has established an excellent rapport. Two options were available to us namely (a) to create a separate independent infrastructure exclusively for the trial, and (b) to work in close cooperation with the State Government's leprosy control programme.

The creation of a separate infrastructure for the vaccine trials had the advantage of 
having the entire staff under one command, but this was outweighed by the numerous disadvantages: (i) a preparatory phase of a few years would be required for training the staff and for carrying out a fresh survey to gather baseline information; (ii) it would take many years to establish a proper rapport with the community, without which no programme would have any chance of success; and (iii) the study would have been expensive.

We therefore preferred the second option and worked in collaboration with the existing health infrastructure of the State Government in the trial area. Though this approach posed an apparent problem of dual administrative control, it was due to this very approach that the large scale trial could be launched within a few months of receiving the financial grant. The collaboration also provided access to readily available epidemiological data on leprosy.

\section{Study design}

\section{OBJECTIVES}

The objective of the trial is to find the relative efficacy of ICRC vaccine over BCG, in terms of lowering the incidence of leprosy in healthy household contacts (HHC) of leprosy patients. The trial is double blind, randomized and controlled. Leprosy is diagnosed and classified as per Indian Consensus classification ${ }^{7}$ with a modification that borderline leprosy has been further divided into borderline tuberculoid and borderline lepromatous. Accordingly, in this trial, leprosy is classified into 6 types namely indeterminate, tuberculoid, borderline tuberculoid, borderline lepromatous, lepromatous and pure neuritic.

The choice was between (i) a study of absolute efficacy of the ICRC vaccine in which the vaccine or a placebo would be administered to eligible volunteers; and (ii) a study to determine the comparative efficacy of ICRC vaccine versus an existing mycobacterial vaccine $(\mathrm{BCG})$ in reducing the incidence of leprosy.

The first option would be, scientifically, the best approach and fewer numbers of volunteers would have to be vaccinated, since the placebo offers zero protection. However, ethically speaking, no eligible volunteer should be deprived of the beneficial effects, if any, of a vaccine. Moreover, the trial would not be strictly double blind unless the 'control' causes an identical local response.

The second option would not provide information on the absolute efficacy of the vaccine but had the advantage that the trial would be strictly double blind because the local response induced by both vaccines would be identical. BCG vaccination is a national programme. BCG itself has given varying protection against leprosy in different trials. ${ }^{8,9}$ A candidate leprosy vaccine should give substantially higher protection than BCG if it has to be adopted as a national modality for leprosy immunoprophylaxis, that is why we chose the second option and used BCG as the control arm of the trial.

The capacity of a vaccine to lower the incidence of a disease is the acid test of its efficacy. Therefore in the trial this was the only parameter used. No attempt was made to measure the immunological parameter(s). The idea of subjecting the HHC to laboratory immunological procedures, even in a subset of vaccinees, was rejected, since it would have created an impression of preferential treatment to certain groups in the population. Also in general, any invasive investigation would result in non-compliance. 


\section{THE TARGET POPULATION}

As mentioned below, under field conditions there is often overdiagnosis. Therefore, to be certain only $\mathrm{HHC}$ of patients exhibiting active lesions at the time of intake in the trial were vaccinated. A household is defined as a group of persons who live together and have their main meals prepared in the same kitchen. The HHC constitute a high risk group. ${ }^{10}$ They are aware about the disease and are already motivated to accept possible prophylactic measures against leprosy. Since the HHC include both lepromin positive and negative individuals, this group will be a sub-set of the general population. Therefore the results of this trial could be generalized to the entire population. But this approach has the disadvantage that a large population would have to be covered over a vast geographical area to get the required number of eligible HHC.

Multidrug therapy (MDT) was introduced in late 1987 in 7 hyperendemic districts of Maharashtra State including the 3 districts selected for the vaccine trial. In this WHOsponsored study, MDT is given only to leprosy patients diagnosed as 'active' by specially trained doctors. The initiation of MDT in the trial area introduced another variable because MDT, for even a short period, is known to 'cure' leprosy or clear up early lesions of leprosy. Therefore, after July 1988, vaccine has been given to eligible HHC of all those leprosy patients who are on WHO-sponsored MDT. However, a separate record is kept of those patients on MDT who are clinically active and inactive, at the time of vaccination.

Among the $\mathrm{HHC}$, one of the possible approaches would be to vaccinate only the HHC of $\mathrm{MB}$ leprosy patients, since it is recognized that they run the highest risk of contracting the disease. The relative risk being 7 times that observed in a non-contact. ${ }^{10}$ This approach has been rejected because: (a) the number of $\mathrm{MB}$ patients is declining fast because of early detection and treatment, and (b) lepromatous rate in dark skinned populations is low. Consequently, an enormously large population would have to be covered over a wide area to get the requisite number of $\mathrm{HHC}$. This would pose problems of logistics and communication. Moreover, it is accepted that non-lepromatous patients can not only transmit leprosy, but the relative risk is higher than the non-contacts.

\section{STATISTICAL ASPECTS}

According to the State Health Authorities for Leprosy Control, the average annual incidence of all forms of leprosy in the HHC is 4-5 per 1000 per year. Through a quick sample survey, it is observed that there is often over-diagnosis to the extent of $20-30 \%$. Therefore, the overall incidence is assumed to be 3.5 per 1000 per year for estimating the size of the trial population. BCG coverage in the trial area, as judged by the presence of a $\mathrm{BCG}$ scar, is about $30 \%$. As mentioned earlier, $\mathrm{BCG}$ has given varying protection against leprosy in various trials. A protection of $25-30 \%$ was observed in the BCG trial at Madras (India). ${ }^{9}$ This is also considered to be the likely protection offered by BCG in the trial area. Presuming a vaccine efficacy of $33.3 \%$ over that of BCG for a $5 \%$ level of significance (one-tailed test), power of significance $90 \%$ and $30 \%$ losses during follow-up, the sample size for the two-arm trial with a 5-year follow-up would be about 31,000 (Table 2). A vaccine with an efficacy of lower than $33.3 \%$ would only be of academic interest. This means that with an average of 4 contacts per household (the national average), the household contacts of 8000 to 10,000 leprosy patients would have to be vaccinated. The 
Table 2. Sample size for the trial (with $30 \%$ BCG coverage)

${ }^{x} 1-$ tail $=0 \cdot 05$.

$1-\beta=0 \cdot 90$.

\begin{tabular}{lccc}
\hline $\begin{array}{l}\text { Follow-up } \\
\text { period }\end{array}$ & $\begin{array}{c}\text { Mean } \\
\text { incidence }\end{array}$ & $\begin{array}{c}\text { Assumed vaccine } \\
\text { efficacy of 33.3\% } \\
\text { over BCG }\end{array}$ & $\begin{array}{c}\text { Assumed vaccine } \\
\text { efficacy of } 50 \% \\
\text { over BCG }\end{array}$ \\
\hline $\begin{array}{l}2 \text { years } \\
5 \text { years }\end{array}$ & $8 \cdot 75$ & 66,262 & 25,636 \\
\hline
\end{tabular}

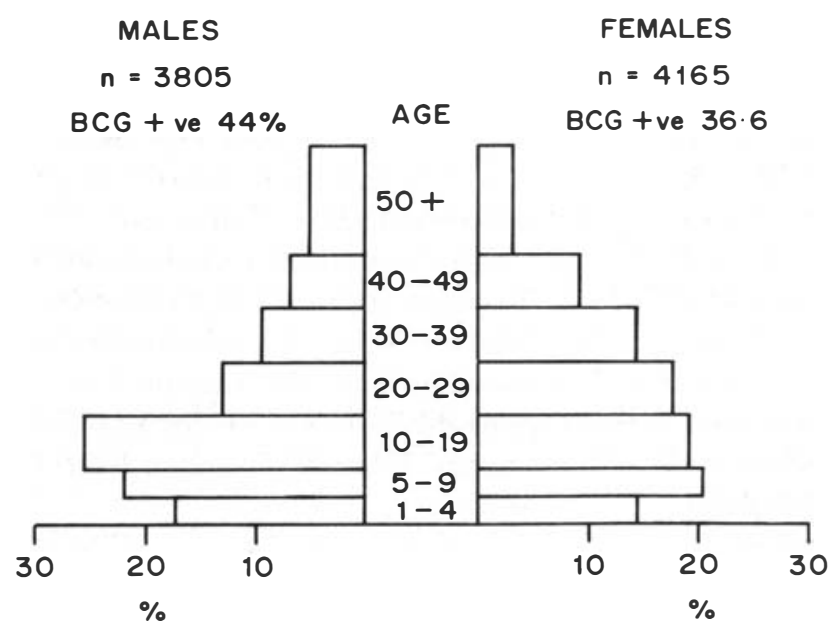

Figure 2. Age distribution of healthy household contacts.

age distribution of the target population based on the analysis of 7960 vaccinees is shown in Figure 2.

\section{RANDOMIZATION}

All human trials would have limitations for operational and ethical reasons, and are thus unlikely to be perfect. Design of the trial always takes into account variables and constraints likely to influence the outcome of the trials. However, some unforeseen variables may crop up during the course of the trial. It is essential that all the variables, known and unforeseen, should equally affect both study and control groups. This can be assured only by proper randomization which was achieved using the following procedure.

The vials containing ICRC and BCG vaccines are randomly assigned and so are their positions (left or right) in the satchel. In the field the eligible volunteers among the HHC are registered as they come (randomly). During vaccination, the first eligible HHC in the household receives the vaccine from the left side of the satchel. The second $\mathrm{HHC}$ receives vaccine from the right side of the satchel. Other contacts receive the vaccine alternatively from the left or right side of the satchel. When the vaccine in one of the 2 vials is finished, 
the pair is discarded even if the other vial contained vaccine. A new pair is used and vaccination starts with the vial in the left side of the satchel.

The 'blindness' of the trial is ensured as follows: (i) identical coded vials are used for the ICRC and BCG vaccines; (ii) both the vaccines are randomly paired; (iii) a separate double blind preliminary study was done to ensure that both vaccines induce identical local response; and (iv) information on the type of vaccine used (codes) will not be available to the field units. The codes are kept at the central headquarters of the project at Bombay.

\section{Biological products}

Vaccines used in the trial are: (i) ICRC vaccine $\left(0.5 \times 10^{9}\right.$ gamma-radiation killed organisms per dose); (ii) BCG vaccine obtained from BCG Laboratories, Madras (India). Standard dose consists of $0 \cdot 1 \mathrm{mg}$ moist weight of $\mathrm{BCG}=2-4 \times 10^{5}$ colony forming units of Danish strain 1331. An entire batch (No. 838) has been kept aside for the trial. Both vaccines are administered intradermally in the right deltoid region and produce a comparable local reaction consisting of delayed inflammation and ulceration that heals in 4-6 weeks. It is not advisable to inject the full dose of BCG in the already sensitized Indian population, since it could produce untoward reactions. BCG therefore is given as $1 / 5$ of the standard dose. This dose was selected on the basis of preliminary studies. At this level the local reaction is similar to that induced by the ICRC vaccine. The vaccines are carried to the villages in vaccine carriers (approved by UNICEF for Expanded Programme of Immunization) and are used within 6 hours. Unused vaccines are returned to the Project Office for further disposal.

\section{Operational aspects}

A field unit has been established at Solapur city, with office and laboratory space. This city is $428 \mathrm{~km}$ from Bombay and is well connected by railways and road. A sub-station was subsequently established at Latur city, about $150 \mathrm{~km}$ away from Solapur. Both offices have facilities for maintaining the cold chain for the vaccines. The vaccine is brought from the Cancer Research Institute, Bombay in dry ice and is immediately transferred to a deep freezer in the project office at Solapur. It is stored in similar condition in the sub-station at Latur. BCG vaccine is reconstituted on the day of use.

Before starting the vaccination in a new administrative unit ('taluka'-equivalent to a county) all aspects of the vaccine project, including the design of the trial, are discussed with local health authorities at various levels. Special meetings are held with LCU staff. A small public function in which local leaders, media-men and members of the community, including students, participate, is held to create awareness in the population. Similar meetings are held at the village level. At the meetings all aspects of the trials are discussed including possible side-effects of the vaccine. Households are told that their participation is entirely voluntary; no incentives are offered and no coaxing is done. They are assured that any problem would be promptly attended to.

Each vaccine team consists of a doctor, trained male and female paramedical personnel from the project staff and one LT and a medico-social worker. On the day of 
vaccination, the vaccine team reaches the village before 7.00 am by which time most village folk are awake. The sunrise in the trial area is approximately $5.45 \mathrm{am}$ and $6.30 \mathrm{am}$ in summer and winter respectively. The doctor meets the village leaders, who send their representative with the vaccine team. On the basis of the list of patients provided by the LCU, the patients are visited on a house-to-house basis. The head of the household is told by the doctor about the vaccines. The simultaneous survey, clinical examination and vaccination are completed before $10 \mathrm{am}$, by which time the villagers leave for work. On average, about 4-6 families are examined and 20-30 HHC vaccinated by one team every day. There are four teams and they vaccinate about 20 days in a month.

The vaccinees are given a pamphlet which explains clearly, in the local language, the sequence of events following vaccination which is similar to that of the BCG vaccine. Being an ongoing national programme the villagers are well aware of the sequence of events following BCG vaccination. This makes acceptance of the ICRC vaccine easy.

The HHC are thoroughly clinically examined by the doctor for evidence of leprosy. The presence of BCG scar is also recorded. Female HHC are examined with the help of the trained female paramedical worker in the team. HHC with suspicious signs of leprosy are excluded from the trial. Simultaneous to the clinical examination, the vaccines are randomly given in the right deltoid region. Regular BCG vaccination in childhood is given on the left deltoid region in India. The scar due to the vaccines used in this trial would remain a permanent evidence of vaccination on the right deltoid region. Since no other vaccine is given in this part of the body, there would be no problem of identifying volunteers during the follow-up phase.

Only HHC of both sexes, between 1 and 65 years of age participate in the study. Pregnant women and persons with chronic debilitating diseases, severe malnutrition, history of allergic reactions, epilepsy and tuberculosis are excluded from the trial.

\section{Immediate follow-up}

The team visits the vaccinees periodically to remove any apprehension in case of minor side-effects. A follow-up protocol has been so designed that it covers all the major sequence of events in the take-up of the vaccine and the protocol can be completed by any trained paramedical worker. The immediate follow-up visits helped us quickly establish rapport with the vaccinees. In addition to the follow-up visits, a system of feedback through postal intimation has been devised for the benefit of vaccinees who may be absent during the visits. The vaccinees are given the address of the Project Office to which they may write or are advised to contact the Medical Officer of the nearest Primary Health Centre. The address of the Project Office is printed on the above mentioned pamphlet and illiterate vaccinees are requested to seek the help of a literate person to write to the Project Office. A system of feedback from the local health workers was also devised. The health workers are advised to inform postally or in person (whichever was more convenient). The feedback system is so effective that even minor problems (usually secondary infection at the vaccination site) are promptly attended to. If the family is poor, a blank postcard is given so that the vaccinee may again inform about his condition. If the vaccinee prefers to visit the office in person, he/she is immediately given the return bus fare on producing the bus ticket. This system of re-imbursement prevented any financial loss to the vaccinee. 


\section{Sociocultural aspects}

Successful implementation of field trials requires the compliance of the eligible volunteers, which in turn depends on the tolerance of the community towards the disease. Based on the experience from the pilot study at Palghar and Satpati, ${ }^{4}$ it was anticipated that the following factors may result in non-cooperation and non-compliance: (a) fear or stigma about leprosy; (b) ignorance about the disease; (c) lack of motivation; (d) disturbance in daily routine; and (e) fear of injectables and invasive techniques.

But the above-mentioned problems did not crop up since we took sociocultural aspects into consideration in the study design and operational strategy. We recognized that the village leaders are highly respected and that their support is essential for the success of any programme. We make it a policy to explain to them the facts about the vaccine. Assistance from the village leaders also ensures community cooperation. India has a male dominated society and it is usually the father who decides all matters including acceptance of prophylactic measures. For these reasons working through a senior male member of the family is highly effective.

Even in a relatively urbanized district like Solapur, the overall literacy rate is barely $40.68 \%$ (Table 1). Therefore we had decided to educate the target groups in the community. Each member of the vaccine team is trained in health education and the doctors accompanying the team are postgraduates in community medicine.

Linguistically, Akkalkot taluka of Solapur district has a large number of Kannada speaking people while Osmanabad and Latur districts are predominantly Marathi speaking. The team members know Marathi (the official state language) and make a special effort to learn a few terms used in daily parlance in colloquial Kannada.

Since it is a common practice to use audio-visual aids for health education, we made a film on the vaccine which was found useful for discussions with healthy personnel. Subsequently, we learnt that at the level of the community a personal approach in communication scored over 'hi-tech' audio-visual aids, so we stopped screening the film at community level. Our experience is that mass media and audio-visual aids are only a supplement, and not a substitute, to a person-to-person approach.

The services of the doctor in the primary health centre are usually clinic-based and principally curative. Doctors seldom visit each house for delivery of preventive services like immunization which is mostly done by paramedical workers. The presence of doctors in our vaccination teams increased the confidence of target groups and improved compliance. Our approach also involved treatment of minor ailments which came to our notice while vaccinating. It has been our experience that, to be more effective, all preventive services should be backed by curative services (at least for minor ailments) by the same team.

Repeated follow-up of vaccinees and feedback from vaccinees by postal intimation and prompt treatment of any problem removed apprehensions and instilled confidence in the target groups. The target groups soon realized that the vaccine is rather innocuous, does not interfere with daily routine and that any problems are promptly attended to. These factors are responsible for the very high compliance of $95 \%$. There are minor variations in compliance in various sociocultural and linguistic groups which are not statistically significant to be mentioned here. Since our teams reached the village early in the morning, about $90 \%$ of the permanently resident (enumerated) HHC were covered. The vaccine is well received in neighbouring villages probably because such news spreads by word of mouth. 


\section{References}

1 Deo MG, Bapat CV, Bhalerao V, Chaturvedi RM, Bhatki WS, Chulawalla RG. Anti-leprosy potentials of ICRC vaccine: a study in patients and healthy volunteers. Int $J$ Lepr, 1983; 51: 540-9.

2 Bhatki WS, Chulawalla RG, Bapat CV, Deo MG. Reversal reaction in lepromatous patients induced by a vaccine containing killed ICRC bacilli-a report of five cases. Int $J$ Lepr, 1983; 51: 466-72.

3 Deo MG. Leprosy Vaccines. Proc. International Symposium on Nuclear Techniques in Parasitic and Communicable Diseases held in Bombay from 21-25 November 1988. Int. Atomic Energy Agency, Vienna, 1988 (In press).

${ }^{4}$ Chaturvedi RM, Chirmule NB, Yellapurkar MV, Shaikh SU, Deo MG. Effects of ICRC anti-leprosy vaccine in healthy subjects. Int $J$ Lepr, 1987; 55: 657-66.

5 Godal T. Immunological aspects of leprosy-present status. Prog Allergy, 1978; 25: 211-42.

6 Waters MFR, Turk JL, Wemambu SNC. Mechanisms of reaction in leprosy. Int J Lepr, 1971; 39: 417-28.

7 Thangraj RH. A manual of leprosy. 5th ed. (1987) Printaid, New Delhi, India (1987) pp 92-105.

8 Noordeen SK. BCG vaccination in leprosy. Develop Biol Standard, 1986; 58: 287-92.

9 Tripathy SP. BCG trial in leprosy. Int J Lepr, 1984; 56: 686-7.

10 Newell KW. An epidemiologist's view of leprosy. Bull WHO, 1966; 34: 827-57. 